EPJ Web of Conferences 41, 08009 (2013)

DOI: $10.1051 /$ epjconf/20134108009

(C) Owned by the authors, published by EDP Sciences, 2013

\title{
Coherence dynamics in light-harvesting complexes with two-colour spectroscopy
}

\author{
Gethin H. Richards ${ }^{1}$, Paul M. G. Curmi ${ }^{2}$, Krystyna E. Wilk ${ }^{2}$, Harry M. Quiney ${ }^{3}$ and Jeffrey A. Davis ${ }^{1}$ \\ ${ }^{1}$ Centre for Atom Optics and Ultrafast Spectroscopy, Swinburne University of Technology, \\ Melbourne, Victoria, Australia \\ ${ }^{2}$ School of Physics and Centre for Applied Medical Research, St. Vincents Hospital, The University \\ of New South Wales, Sydney, New South Wales, Australia \\ ${ }^{3}$ School of Physics, The University of Melbourne, Victoria, Australia
}

\begin{abstract}
We investigate coherent dynamics in the cryptophyte light-harvesting complex Phycocyanin-645 (PC-645). A two-colour four-wave mixing experiment allows us to isolate a coherence pathway and observe its evolution in the absence of other signals. We measured a decoherence time of $540 \mathrm{fs}$ for the coherence [1]. Additionally oscillations in the signal pathway give evidence for the coherent excitation of states outside the bandwidth of the laser pulse. This suggests strong coupling between the excited states and phonon modes [1].
\end{abstract}

\section{Introduction}

Long-lived coherences, that persist for time-scales relevant to energy transfer, have been demonstrated in light-harvesting complexes using two-dimensional spectroscopy experiments [2,3]. These coherences were attributed to beating between the excitonic eigenstates of the system, leading to much speculation on their possible role in energy transfer in the system. In broadband twodimensional spectroscopy multiple states of the light-harvesting complex are excited simultaneously. This means that the two-dimensional spectra are typically very congested with numerous population and coherence pathways overlaid with one another. Extracting details on the coherent system interactions becomes difficult in this environment. To gain more insight into these light-harvesting complexes we have developed a two-colour photon echo spectroscopy.

The light-harvesting complex we investigate is Phycocyanin-645. The structure of the complex is known to a high-resolution and shows 8 separate chromophore molecules held in a protein scaffold [4]. Two of the chromophoric dihydrobiliverdin (DBV) molecules at the centre of the complex form a strongly coupled excitonic dimer, at the centre of the complex. These states are known as DBV+ the higher energy state and DBV- the lower energy state [4]. Another two chromophore molecules at the periphery are called mesobiliverdin (MBV) and also absorb along the blue edge of the absorption spectrum. Previous two-dimensional experiments has identified the presence of coherence between the DBV+ and MBV states [2,3].

This is an Open Access article distributed under the terms of the Creative Commons Attribution License 2.0, which permits unrestricted use, distribution, and reproduction in any medium, provided the original work is properly cited. 


\section{Experiment}

In our two-colour photon echo spectroscopy, the first two pulses, referred to as $\mathbf{k}_{1}$ and $\mathbf{k}_{\mathbf{2}}$, are tuned to $2.18 \mathrm{eV}$ and $2.06 \mathrm{eV}$, and excite a coherent superposition of states. The energies of the pulses are chosen to be resonant with the expected spectral position of the DBV+ and MBV excitonic states. These pulses have a bandwidth of approximately $17 \mathrm{meV}$ (or $15 \%$ of the energy separation between the pulses), which eliminates the possibility that population pathways will contribute to the signal. They are separated by a time delay called the coherence time, $\tau$. A third pulse, $\mathbf{k}_{\mathbf{3}}$, interacting with the system, after a time delay $\mathrm{T}$ (called the waiting time) induces a third-order non-linear polarization in the phase matched direction. This signal is defined as $\mathbf{k}_{4}=-\mathbf{k}_{1}+\mathbf{k}_{2}+\mathbf{k}_{3}$. We measure the spectrally resolved intensity of this signal as a function of the two time delays. The signature of coherence in this experiment will be any signal $\mathbf{k}_{4}$ which persists for longer than the pulse overlap. The experiment is performed at $77 \mathrm{~K}$.

\section{Results and Discussion}
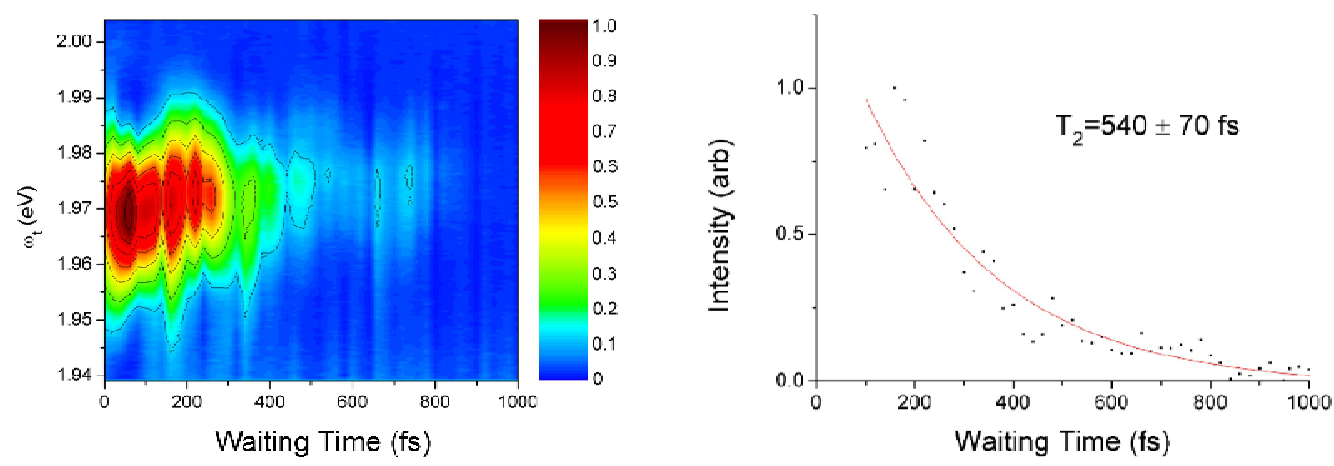

Fig. 1. The spectrally resolved signal intensity of $\mathbf{k}_{4}$ is shown as a function of the waiting time delay. To the right is signal integrated across the spectral domain. Modified from [1].

Figure 1 demonstrates signal persisting for longer than $800 \mathrm{fs}$ and offers direct evidence for coherence between DBV+ and MBV. By integrating across the spectral domain a dephasing time of $540 \mathrm{fs}$ is measured for this coherence.

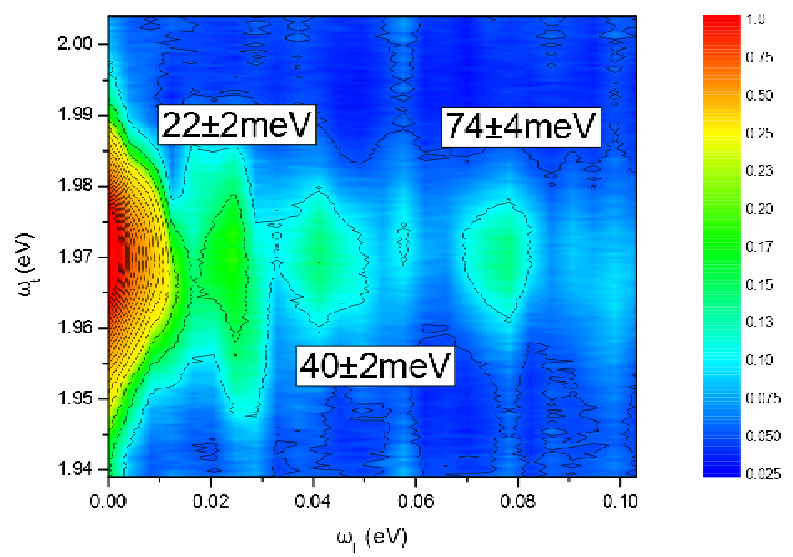

Fig. 2. The Fourier transform across the time domain shows three individual beats responsible for the oscillations observed in the temporal domain. The peaks, which are reasonably well separated, appear at $22 \pm 2 \mathrm{meV}, 40 \pm 2 \mathrm{meV}$ and $74 \pm 4 \mathrm{meV}$. Modified from [1]. 
The oscillations observed in experiment are not due to polarization interference [1]. Additionally the experiment will not be sensitive to the type of coherent oscillations identified by previous two-dimensional spectroscopy, as we are measuring the intensity rather than electric field. If however in the experiment there is excitation of an additional state, in phase with one of the incident laser pulses, interference between two quantum pathways will cause quantum beating. By taking the Fourier transform across the waiting time domain we see three clear components in Fig. 2 which cause the oscillations in the signal. This offers an explanation for the $22 \mathrm{meV}$ peak as it is very close to the bandwidth of the pulse used in experiment. If one of the pulses coherently excites two states separated by $22 \mathrm{meV}$, interference between the two signal pathways will cause beating at that energy.

The peaks at $40 \mathrm{meV}$ and $74 \mathrm{meV}$ imply that some other state or states are being excited. As the energies of the beating are far outside the bandwidth of the pulse, another mechanism must be exciting this state(s). We can infer that this process must be coherent in order for us to see the quantum beating. We suggest that the absorption or emission of a coherent phonon must be involved in exciting this additional state. The experiment however is unable to distinguish precisely the mechanism for exciting this additional state. One possibility is that phonon assisted absorption excites an additional state. Another is that there is an inherent mechanism in this light-harvesting complex whereby the energy is coherently transferred between states. This mechanism would also need to involve coherent phonon modes. Although we are unable to distinguish between these possibilities, the coherent excitation of this intermediate state implies that phonon modes are strongly coupled to the excited states.

Previous work has shown a state, labelled DBV-, at $2.11 \mathrm{eV}$. The energy difference between this state and those directly excited in the experiment are in remarkable agreement with the energies identified by the quantum beating, strongly suggesting the involvement of this DBV- state. Further work exploring the dynamics of the $40 \mathrm{meV}$ and $74 \mathrm{meV}$ beats confirms that this beating occurs in the excited state manifold and adds further support to this state attribution [5].

In conclusion we have demonstrated that the two-colour experiment is a versatile method for observing coherences in light-harvesting complexes. The advantage of the technique is that coherence pathways can be observed in isolation from competing signals. It has been shown that coherence between DBV+ and MBV, in PC645, has a dephasing time of 540fs. The energies and dynamics of the observed quantum beating give a strong indication that an additional state is excited in experiment and this is the DBV- state, in the excited state manifold. The coherent excitation of this state provides evidence for strong coupling to coherent phonon modes.

\section{References}

[1] G H Richards, K E Wilk, P M G Curmi, H M Quiney and J A Davis; J. Phys. Chem. Lett. 3272 7 (2012)

[2] E Collini, C Y Wong, K E Wilk, P M G Curmi, P Brumer and G D Scholes; Nature 463 644-8 (2010)

[3] D B Turner, K E Wilk, P M G Curmi and G D Scholes; J. Phys. Chem. Lett. 2 1904-11 (2011)

[4] T Mirkovic, A B Doust, J Kim, K E Wilk, C Curutchet, B Mennucci, R Cammi, P M G Curmi and G D Scholes; Photochem. Photobiol. Sci., 6 964-75 (2007)

[5] G H Richards, K E Wilk, P M G Curmi, H M Quiney and J A Davis; J. Phys. B. In Press (2012) 